\title{
Influencia de diferentes productos inoculantes sobre el poder de nucleación y la tendencia a la contracción de la fundición con grafito esferoidal $\left(^{(*)}\right.$
}

\author{
R. Suárez*, J. M. Gutiérrez*, A. Loizaga*, P. Larrañaga* y J. Sertucha*
}

Resumen

\begin{abstract}
La utilización de sistemas de control metalúrgico, basados en el análisis de las curvas de solidificación, permite examinar las características del metal a lo largo de todo el proceso de fabricación. En este trabajo, dichos sistemas se han utilizado con el fin de estudiar la eficacia de la etapa de inoculación del metal, determinar qué substratos ofrecen mejores resultados a la hora de nuclear el grafito esferoidal, minimizar la tendencia a la formación de cementita y reducir al máximo la tendencia a la contracción de la aleación en la etapa final de la solidificación. Con este objeto, se han seleccionado 10 inoculantes comerciales, con diferencias en su composición química, con el propósito de obtener información sobre la eficacia de los elementos químicos utilizados comercialmente para germinar núcleos grafíticos en la fundición esferoidal. El estudio se completa con pruebas sobre pieza real, donde se verifican los resultados obtenidos con los inoculantes seleccionados.
\end{abstract}

Palabras clave Poder de nucleación; Tendencia a la contracción; Fundición con grafito esferoidal; Inoculante; Solidificación; Análisis térmico.

\section{Influence of different inoculants on the nucleation potential and the contraction ability of the ductile iron}

\begin{abstract}
The use of advanced control systems based on cooling curves allows to analyse the characteristics of the melt in real time along the manufacturing process. In this work, the system mentioned above has been utilised so as to study the efficiency of the inoculation step, to determine which substrates have the optimum ability for nucleating spheroidal graphite, to minimise the tendency to form cementite phase and to reduce the capacity of the melt to form microshrinkage defects. Thus, 10 different commercial inoculants have been selected in order to investigate the efficacy of several chemical elements included in their composition for nucleating nodules in ductile iron. In addition, trials performed on real castings are included in this work so as to verify the results obtained from the selected inoculants.
\end{abstract}

Keywords

Nucleation potential; Contraction susceptibility; Spheroidal grafite iron; Inoculation; Solidification; Thermal analysis.

\section{INTRODUCCIÓN}

La rentabilidad de los procesos de fabricación en las plantas industriales depende en gran medida de la disponibilidad de técnicas que permitan cuantificar en el menor plazo de tiempo posible la calidad del material. En este sentido, el desarrollo de sistemas experimentales con aplicación en la propia planta y que sean capaces de evaluar las características metalúrgicas del material incluso antes de afrontar la colada de los moldes ${ }^{[1-3]}$ supone una herramienta de gran valor a la hora de minimizar los fallos en la obtención de piezas funcionalmente válidas. En este sentido, se han desarrollado técnicas basadas en el análisis térmico ${ }^{[4}$ y] que son de creciente aplicación industrial ${ }^{[3 \text { y }}{ }^{6]}$ en el campo de la fabricación de piezas de fundición con grafíto esferoidal.

Una de las etapas críticas dentro del proceso de solidificación del hierro de fundición es la formación del grafito como fase heterogénea dentro de la matriz metálica ${ }^{[1}$ y 7 -11]. La forma, distribución y tamaño de estas fases grafíticas influyen, de manera importante, en las características metalúrgicas ${ }^{[12]}$, el tipo de estructura matricial ${ }^{[6}$ y 13$]$ y las propiedades

\footnotetext{
(•) Trabajo recibido el día 3 de octubre de 2008 y aceptado en su forma final el día 23 de enero de 2009.

* Ingeniería y Procesos de Fundición, AZTERLAN. Aliendalde Auzunea n 6, E-48200 Durango (Bizkaia). E-mail: jsertucha@azterlan.es.
} 
mecánicas ${ }^{[14-16]}$ del material seleccionado para fabricar una determinada pieza. Actualmente, las técnicas basadas en el análisis térmico permiten evaluar diversos aspectos críticos dentro del proceso de solidificación de las fundiciones esferoidales. Dos de ellos son el poder de nucleación grafítica (definido por el parámetro $\mathrm{N}$ ) y la capacidad de contracción o tendencia a la formación de microrrechupes (factor k) ${ }^{[3]}$. El cálculo de estos dos parámetros abre un campo de posibilidades a la hora de optimizar diversas etapas en el proceso de elaboración del metal líquido que va a ser colado en los moldes.

La inoculación o aportación de gérmenes de crecimiento grafítico en el seno del metal, todavía líquido, es una etapa de importancia crítica en el proceso de fabricación de piezas de fundición ${ }^{[17}$ y 18] . Esta práctica permite potenciar la capacidad de nucleación del metal para formar agregados grafíticos, normalmente mermada debido a las etapas previas de preparación de la aleación (fusión, mantenimiento, tratamiento con FeSiMg, etc.), mediante la adición de substratos específicos que contienen óxidos, sulfuros y silicatos ${ }^{[7,17}$ y 19]. De este modo, se consigue optimizar la formación de los esferoides grafíticos y evitar en el estado bruto de colada la formación de fases carbúricas en zonas de espesor reducido y/o en aquellas donde existe una elevada velocidad de enfriamiento ${ }^{[17]}$.

Teniendo en cuenta que se produce un desvanecimiento en la capacidad de nucleación, a lo largo del tiempo en el que el metal permanece líquido ${ }^{[17}$ y 18$]$, es necesario considerar el estado o calidad metalúrgi$\mathrm{ca}^{[3]}$ de éste antes de efectuar su inoculación. En este sentido, se establecen dos condiciones industriales de partida: arranque de fabricación y fabricación normal. La primera, corresponde al estado del metal en el período de tiempo inmediatamente posterior al inicio de la fabricación. En estas condiciones, los útiles de proceso (hornos, cucharas y unidades de colada) contienen, todavía, elevados contenidos de oxígeno debido a la interrupción de la actividad de fabricación, por lo que es necesario efectuar tratamientos de esferoidización más intensos con mayores aportes de magnesio y, por lo tanto, la calidad del metal tratado será inferior. La fabricación normal corresponde al desarrollo habitual del proceso sin interrupciones prolongadas durante el mismo.

El presente trabajo analiza la influencia de diferentes substratos, que forman parte de inoculantes comerciales, sobre los procesos de solidificación y las características del metal obtenido tras dicho tratamiento. La evaluación de la efectividad de los inoculantes se realiza en ensayos sobre probeta, comparando los resultados obtenidos a partir de las curvas de solidificación registradas en cada caso y en diferentes condiciones de proceso. Posteriormente, el conocimiento adquirido a partir de estos ensayos se aplica en la fabricación de una pieza real, con el fin de validar los resultados obtenidos. De este modo, se pretende mejorar el conocimiento de los procesos de inoculación y optimizar su efectividad, como paso previo a cualquier tipo de estudio personalizado para cada planta industrial.

\section{PARTE EXPERIMENTAL}

En todos los casos, el metal líquido se preparó con ayuda de tres hornos de inducción de media frecuencia $(250 \mathrm{~Hz})$, con capacidad para 10 t y $500 \mathrm{~kW}$ de potencia. La carga metálica utilizada estaba constituida por un $30 \%$ de paquete de acero procedente del sector de automoción, 20 \% de lingote y el 50 \% de retornos clasificados convenientemente de acuerdo a su composición química. Una vez finalizado el proceso de fusión de la carga en los hornos, se determinaron los contenidos de carbono y silicio con el fin de realizar los ajustes oportunos. El metal líquido finalmente obtenido se preacondicionó mediante la adición del 0,10 \% de un FeSi comercial (\% Si $=75,2$ y \% Al $=1,73)$ para obtener una calidad metalúrgica de partida similar en todas las coladas preparadas.

Antes de proceder al vertido del metal líquido desde los hornos de fusión a una cuchara cilíndrica con capacidad para $2000 \mathrm{~kg}$ y diseñada para realizar los tratamientos de esferoidización siguiendo la metodología tundish-cover, en el interior de una cámara de reacción situada en su parte inferior se introdujeron $22 \mathrm{~kg}$ de la ferroaleación FeSiMg 522 (\% Si = 45,1; \% Mg = 5,2; $\% \mathrm{Ca}=1,7 ; \% \mathrm{Ce}=0,9 ; \% \mathrm{TR}=2,0)$ con ayuda de un tubo direccionador. En condiciones de arranque de fabricación, en los 4 primeros tratamientos se utilizó la ferroaleación FeSiMg 931 (\% Si = 42,2; \% Mg = 9,1; $\% \mathrm{Ca}=2,8 ; \% \mathrm{Ce}=0,8 ; \% \mathrm{TR}=1,1$ ), de forma que se depositaron $25 \mathrm{~kg}$ de este producto en el interior de la cámara de reacción. En todos los casos, el material cubriente utilizado estaba constituido por recortes de acero (tamaño 5-15 $\mathrm{mm}$ ) procedentes de procesos de estampación. El vertido del metal a la cuchara de tratamiento se efectúa cuando la temperatura de éste se encuentra en el intervalo $1.480-1.500{ }^{\circ} \mathrm{C}$. Una vez finalizada la reacción del tratamiento con magnesio, el metal se desescoria convenientemente en la propia cuchara y se traslada a una unidad de colada presurizada.

Los ensayos experimentales incluidos en este trabajo se efectuaron sobre muestras de metal líquido destinadas a la fabricación de piezas de fundición con grafito esferoidal (calidades comprendidas entre la EN GJS 400-15U y EN GJS 500-7). Dichas muestras se obtuvieron a partir del metal contenido en una unidad de colada presurizada con capacidad para $9 \mathrm{t}$ (ref. ABB-CTO5) instalada junto a una línea de moldeo 
vertical Disamatic 230 en la fundición Betsaide, S.A.L. Con el fin de lograr una mayor homogeneidad, la toma de muestra se realizó en la bañera de colada (zona próxima al stopper o sistema de interrupción de la colada) 5 min después del vertido en la unidad de colada del metal procedente de la cuchara de tratamiento.

La tabla I incluye la composición química de los 10 inoculantes comerciales seleccionados inicialmente para llevar a cabo el presente estudio. En todos ellos, la granulometría se adaptó hasta alcanzar un tamaño de partícula de 0,2-0,5 mm con el fin de facilitar su rápida disolución en el metal líquido. Una inspección macroscópica posterior confirmó la inexistencia de impurezas y/o partículas extrañas en cada uno de los productos seleccionados.

El estudio comparativo sobre la influencia de los diferentes inoculantes en el metal de colada se llevó a cabo utilizando la misma muestra en cada una de las series de ensayos realizada. Estas series incluyeron:

- la determinación inicial de la temperatura y la actividad de oxígeno ${ }^{[20]}$ del metal no inoculado y contenido en la unidad de colada,

- la obtención de una muestra destinada a su análisis químico por espectrometría de emisión por chispa,

- la caracterización del proceso de solidificación del metal inoculado con cada uno de los productos incluidos en la tabla I.

De este modo, las series de ensayos se repartieron de igual manera, en 4 días pertenecientes a semanas distintas. Cada día, se inició la actividad en la planta con una serie de ensayos bajo condiciones de arranque (el metal se tomó de la unidad de colada cuando el contenido de magnesio en éste superó el 0,030 \%) y dos series en condiciones normales de fabricación (designadas como X1 y X2, siendo X = 1 a 4). Con el fin de garantizar las condiciones habituales de fabricación, estas dos últimas series se efectuaron transcurridas $4 \mathrm{~h}$ desde el arranque. Así, se obtuvieron 4 series en arranque y 8 series en condiciones normales de fabricación.

En las caracterizaciones térmicas, las muestras de metal obtenidas a partir de la unidad de colada se vierten en el interior de 10 tazas comerciales (Quik-cup ${ }^{\circledR}$ ) que contienen un termopar tipo $\mathrm{K}$ en su interior y una determinada cantidad de uno de los inoculantes seleccionados para este estudio. La adición del inoculante en las tazas fue del 0,15\% en condiciones de fabricación normal y 0,20 \% en arranque, siguiendo la instrucción establecida en la planta para evitar la formación de carburos en las condiciones metalúrgicas más desfavorables. Estos procesos de inoculación aumentan el contenido de silicio, aproximadamente, un $0,1 \%$ en el metal de colada. La recogida y tratamiento de los datos obtenidos en todos los ensayos de análisis térmico se llevó a cabo con ayuda del sistema de gestión metalúrgica Thermolan ${ }^{\circledR[3]}$. La caracterización de las curvas registradas y la determinación de los parámetros $\mathrm{N}$ y k se efectuó con ayuda de un software especialmente diseñado para tal efecto e integrado en este sistema de gestión metalúrgica. El intervalo de temperatura utilizado para registrar las curvas de solidificación de todas las muestras analizadas térmicamente es $1.210-1.050^{\circ} \mathrm{C}$. En esta zona se detecta eficazmente la transición eutéctica. En cada caso, el vertido de metal en las tazas se realizó de manera que éstas queden llenas sin rebosar metal hacia el exterior (aproximadamente 330-350 g de metal). Posteriormente, el sistema se mantuvo en condiciones estables durante el período de recogida de datos por parte del termopar.

Tabla I. Composiciones químicas de los inoculantes seleccionados ( $\%$ en peso)

Table I. Chemical compositions of the selected inoculant products (wt. \%)

\begin{tabular}{lcccccccccc}
\hline \multicolumn{1}{c}{ Inoculante } & $\mathbf{S i}$ & $\mathbf{A l}$ & $\mathbf{C a}$ & $\mathbf{B i}$ & $\mathbf{C e}$ & $\mathbf{T R}^{*}$ & $\mathbf{Z r}$ & $\mathbf{B a}$ & $\mathbf{M n}$ & $\mathbf{S}$ \\
\hline INOC1 & 76,9 & 0,83 & 0,79 & - & - & 1,83 & - & - & - & 0,007 \\
INOC2 & 75,1 & 0,89 & 0,93 & - & 1,70 & - & - & - & - & 0,110 \\
INOC3 & 67,1 & 1,47 & 1,06 & - & - & - & 3,01 & - & 3,06 & 0,005 \\
INOC4 & 73,1 & 0,71 & 1,75 & 0,88 & - & 0,62 & - & - & - & 0,006 \\
INOC5 & 75,9 & 1,17 & 2,46 & - & - & - & 1,03 & - & - & 0,007 \\
INOC6 & 63,3 & 0,83 & 1,63 & 0,35 & 0,11 & 0,97 & - & 0,15 & - & 0,006 \\
INOC7 & 63,0 & 0,81 & 1,62 & 0,34 & - & 0,87 & - & 0,19 & - & 0,007 \\
INOC8 & 72,8 & 1,40 & 0,81 & - & - & - & - & 0,60 & 0,21 & 0,004 \\
INOC9 & 68,2 & 1,45 & 1,61 & - & 1,05 & 0,71 & - & - & 0,24 & 0,005 \\
INOC10 & 68,8 & 1,30 & 0,85 & - & - & - & 1,35 & 0,24 & 1,32 & 0,004 \\
\hline
\end{tabular}

*La y cantidades minoritarias de otros elementos del grupo de las tierras raras. 
En las series de arranque 1 y 3 , y en las series obtenidas bajo condiciones normales de fabricación 11 y 31 , se llevaron a cabo ensayos para evaluar la tendencia del metal a la formación de carburos bajo condiciones de enfriamiento rápido. Así, de forma paralela a la realización de los ensayos de caracterización térmica y química, el metal obtenido a partir de la unidad de colada se vierte en 10 moldes fabricados específicamente para obtener cuñas de temple (Fig. 1). Cada uno de estos moldes contenía el 0,15 \% (fabricación normal) ó 0,20 \% (arranque) del producto inoculante seleccionado en cada caso. Inmediatamente después de la colada de los moldes, éstos se rompen y la cuña contenida en su interior se introduce en un baño de agua con agitación hasta lograr su enfriamiento a temperatura ambiente. Las cuñas obtenidas se cortan por su parte central y se acondicionan para efectuar su análisis metalográfico. Antes de determinar el
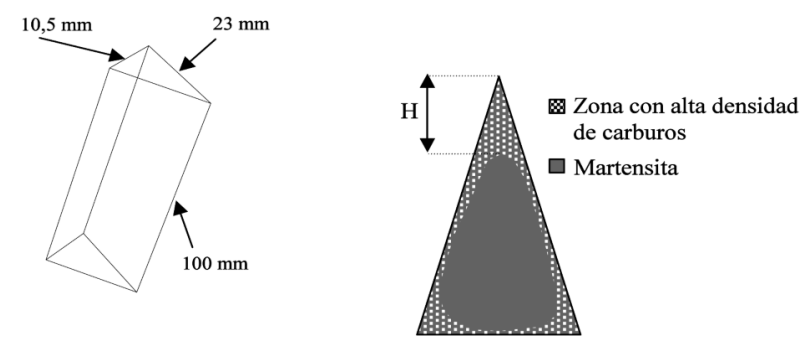

Figura 1. Cuña de temple utilizada para evaluar la tendencia a la formación de carburos.

Figure 1. Chill specimen used for evaluating the carbide formation tendency in the melt. parámetro $\mathrm{H}$ (Fig. 1), se verifica metalográficamente que la inoculación del metal ha sido efectiva.

Las pruebas de fabricación sobre pieza real se efectuaron en una de las 3 líneas de moldeo vertical presentes en la planta. Para ello, se seleccionó una referencia que dispone de 4 huellas en la placa modelo (Fig. 2 a)), siendo las dos pertenecientes al nivel inferior (huellas 3 y 4) las que presentan una zona masiva donde aparece un microrrechupe (Fig. 2 b)). Este defecto se manifiesta como consecuencia de la insuficiencia del sistema de alimentación (o mazarota) a la hora de contrarrestar la contracción del metal en esta zona masiva. Tras el llenado de la unidad de colada con el metal procedente de la cuchara de tratamiento, se fabrican los correspondientes moldes de esta referencia y se cuelan 10, utilizando cada uno de los inoculantes seleccionados. Para asegurar que el sistema automático de inoculación existente en la línea no mezcle los productos inoculantes, antes de las coladas, se aspira el depósito y se adiciona cada uno de los productos seleccionados.

A la vez que se cuelan los 10 moldes utilizando el metal inoculado con cada producto, se lleva a cabo la caracterización térmica de éste siguiendo una metodología similar a la descrita anteriormente. Por otra parte, se toma una muestra de metal inoculado para su análisis químico. Una vez finalizada la colada de los moldes, las piezas se extraen del final de la línea de moldeo, se separan las pertenecientes a la huella $\mathrm{n}^{-} 4$ (considerada históricamente la más crítica) y se inspeccionan por fluoroscopía de rayos $\mathrm{X}$ para determinar la presencia de microrrechupes y cuantificar su volumen. Adicionalmente, en 3 piezas fabricadas con cada inoculante se obtienen muestras para inspección

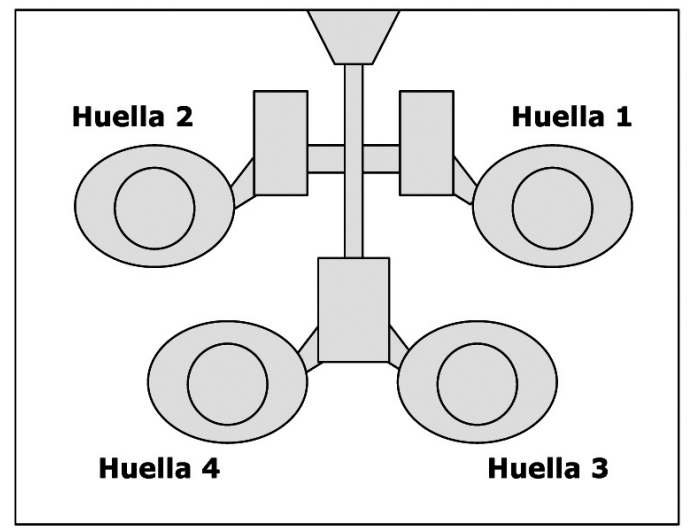

a)

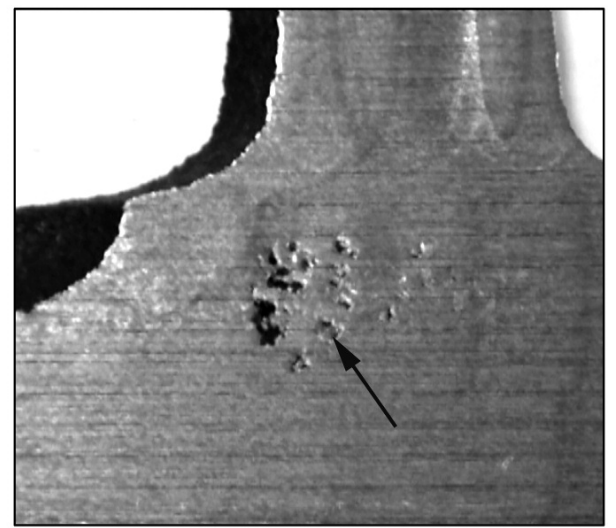

b)

Figura 2. (a) Placa modelo utilizada en las pruebas de fabricación con piezas reales; (b) defecto localizado en una de las piezas.

Figure 2. (a) Pattern plate used for experimental trials on castings; (b) defect found in one of the manufactured castings. 
metalográfica a partir de la zona masiva anteriormente descrita.

\section{RESULTADOS}

La tabla II contiene los resultados obtenidos a partir de los análisis químicos realizados sobre todas las muestras de metal tomadas en la unidad de colada y sin inoculación. En la tabla III, se incluyen los parámetros considerados más significativos en la caracterización térmica y la actividad de oxígeno, medidos sobre el metal no inoculado y utilizado, en cada caso, para llevar a cabo las series de ensayos.
En todos los casos, dicho metal, en condiciones de arranque o fabricación normal, muestra una composición química, temperatura y actividad de oxígeno similares (los dos últimos parámetros son inferiores en condiciones de arranque debido a la baja temperatura de la cuchara y a la mayor intensidad de los tratamientos con FeSiMg). Sin embargo, existen ciertas diferencias en el poder de nucleación del metal sin inoculación, que deben ser atribuidas a variaciones en la influencia que tiene el tratamiento de esferoidización sobre éste. Por ello, el posterior análisis comparativo entre inoculantes debe efectuarse, fundamentalmente, sobre los ensayos pertenecientes a cada una de las series realizadas.

Tabla II. Composición química del metal líquido contenido en la unidad de colada

Table II. Chemical composition of the melt contained in the presspour

\begin{tabular}{|c|c|c|c|c|c|c|c|c|c|}
\hline Serie & Fabricación & C & Si & $\mathrm{Mn}$ & $\mathbf{P}$ & $S$ & Mg & $\mathrm{Cu}$ & $\mathrm{Ti}$ \\
\hline 1 & Arranque & 3,68 & 2,53 & 0,12 & 0,037 & 0,003 & 0,035 & 0,08 & 0,011 \\
\hline 11 & Normal & 3,69 & 2,50 & 0,12 & 0,038 & 0,005 & 0,028 & 0,11 & 0,010 \\
\hline 12 & Normal & 3,70 & 2,55 & 0,13 & 0,038 & 0,004 & 0,029 & 0,09 & 0,009 \\
\hline 2 & Arranque & 3,70 & 2,58 & 0,12 & 0,041 & 0,002 & 0,037 & 0,06 & 0,010 \\
\hline 21 & Normal & 3,72 & 2,66 & 0,11 & 0,040 & 0,006 & 0,030 & 0,03 & 0,010 \\
\hline 22 & Normal & 3,70 & 2,60 & 0,12 & 0,042 & 0,005 & 0,028 & 0,05 & 0,011 \\
\hline 3 & Arranque & 3,72 & 2,54 & 0,11 & 0,039 & 0,003 & 0,036 & 0,12 & 0,012 \\
\hline 31 & Normal & 3,68 & 2,60 & 0,12 & 0,040 & 0,004 & 0,032 & 0,09 & 0,010 \\
\hline 32 & Normal & 3,70 & 2,69 & 0,13 & 0,038 & 0,003 & 0,030 & 0,10 & 0,010 \\
\hline 4 & Arranque & 3,68 & 2,57 & 0,12 & 0,041 & 0,003 & 0,038 & 0,07 & 0,012 \\
\hline 41 & Normal & 3,73 & 2,48 & 0,11 & 0,033 & 0,004 & 0,029 & 0,05 & 0,011 \\
\hline 42 & Normal & 3,67 & 2,53 & 0,11 & 0,039 & 0,004 & 0,033 & 0,07 & 0,011 \\
\hline
\end{tabular}

Tabla III. Caracterización metalúrgica del metal líquido contenido en la unidad de colada

Table III. Metallurgical characterization of the melt contained in the presspour

\begin{tabular}{rccccccc}
\hline Serie & Fabricación & $\mathbf{T}\left({ }^{\circ} \mathbf{C}\right)$ & $\mathbf{T e}_{\text {min }}\left({ }^{\circ} \mathbf{C}\right)$ & $\mathbf{R}\left({ }^{\circ} \mathbf{C}\right)$ & $\mathbf{N}\left(\mathbf{e s f} . / \mathbf{m m}^{2}\right)$ & $\mathbf{k}$ & $\mathbf{A O}(\mathbf{p p m})$ \\
\hline 1 & Arranque & 1.385 & $1.122,1$ & 21,4 & 93 & 0,65 & 0,37 \\
11 & Normal & 1.445 & $1.127,7$ & 17,2 & 136 & 0,69 & 0,54 \\
12 & Normal & 1.435 & $1.129,3$ & 14,7 & 148 & 0,71 & $0,49$. \\
2 & Arranque & 1.372 & $1.119,8$ & 17,3 & 106 & 0,68 & 0,41 \\
21 & Normal & 1.438 & $1.128,7$ & 12,1 & 141 & 0,72 & 0,50 \\
22 & Normal & 1.443 & $1.138,3$ & 7,3 & 189 & 0,76 & 0,47 \\
3 & Arranque & 1.391 & $1.125,6$ & 18,2 & 116 & 0,63 & 0,35 \\
31 & Normal & 1.440 & $1.130,2$ & 9,6 & 147 & 0,71 & 0,56 \\
32 & Normal & 1.448 & $1.126,7$ & 11,9 & 128 & 0,66 & 0,58 \\
4 & Arranque & 1.396 & $1.128,1$ & 13,6 & 124 & 0,67 & 0,40 \\
41 & Normal & 1.445 & $1.127,9$ & 10,5 & 136 & 0,69 & 0,51 \\
42 & Normal & 1.440 & $1.134,4$ & 8,1 & 168 & 0,74 & 0,60 \\
\hline
\end{tabular}


Con el fin de facilitar la comprensión de los resultados obtenidos a partir de todos los ensayos realizados, este apartado se ha subdividido en base a las condiciones de fabricación (arranque o normal), además de las pruebas efectuadas sobre piezas reales.

\subsection{Condiciones de arranque de fabricación}

En las figuras 3 y 4 se representan los valores del pa-

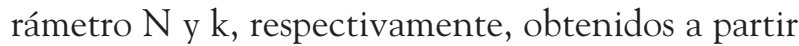
de las curvas de solidificación pertenecientes al metal inoculado con cada uno de los productos comerciales seleccionados para realizar este estudio. En dichas figuras se incluyen las 4 series efectuadas bajo condiciones de arranque.

En relación al poder de nucleación del metal inoculado, se observa que los productos inoculantes INOC8, INOC9 e INOC10 proporcionan los valores más bajos del parámetro $\mathrm{N}$ en las 4 series de ensayos realizadas. Entre ellos, el INOC8 produce las muestras de metal inoculado con menor capacidad de nucleación. Aunque el resto de inoculantes muestran una efectividad nucleadora del grafito comparable, el producto INOC2 destaca ligeramente sobre el resto en, prácticamente, todas las series analizadas.

El comportamiento observado en la capacidad de las muestras inoculadas para contraer durante la fase final del proceso de solidificación es similar al descrito para el poder de nucleación. Los inoculantes con menor poder de formación de núcleos grafíticos (INOC9, INOC10 y especialmente el INOC8) también dan lugar a muestras de metal con valores más reducidos del parámetro $\mathrm{k}$, es decir, mayor tendencia a la formación de microrrechupes (Fig. 4). Nuevamente, el caso más desfavorable corresponde al producto INOC8. Dentro de los casos propicios, no parece haber un inoculante destacable entre el resto de productos analizados.

La tabla IV muestra los resultados de los ensayos efectuados para evaluar la tendencia del metal inoculado a la formación de carburos. En condiciones de arranque, los productos INOC8, INOC9 e INOC10 originan los valores más elevados del parámetro $\mathrm{H}$ en las dos series de ensayos.

\subsection{Condiciones de fabricación normal}

Las figuras 5 y 6 muestran la evolución de los parámetros $\mathrm{N}$ y k para las diferentes muestras inoculadas en condiciones normales de fabricación. En las 8 series de ensayos llevadas a cabo en este estado se obtienen valores del parámetro $\mathrm{N}$ sensiblemente inferiores a los obtenidos en los arranques. Este hecho parece estar relacionado con la menor adición de inoculante al metal en condiciones normales $(0,15 \%)$. No ocurre lo mismo en el caso del parámetro k, donde se obtienen evoluciones similares en ambas condiciones de

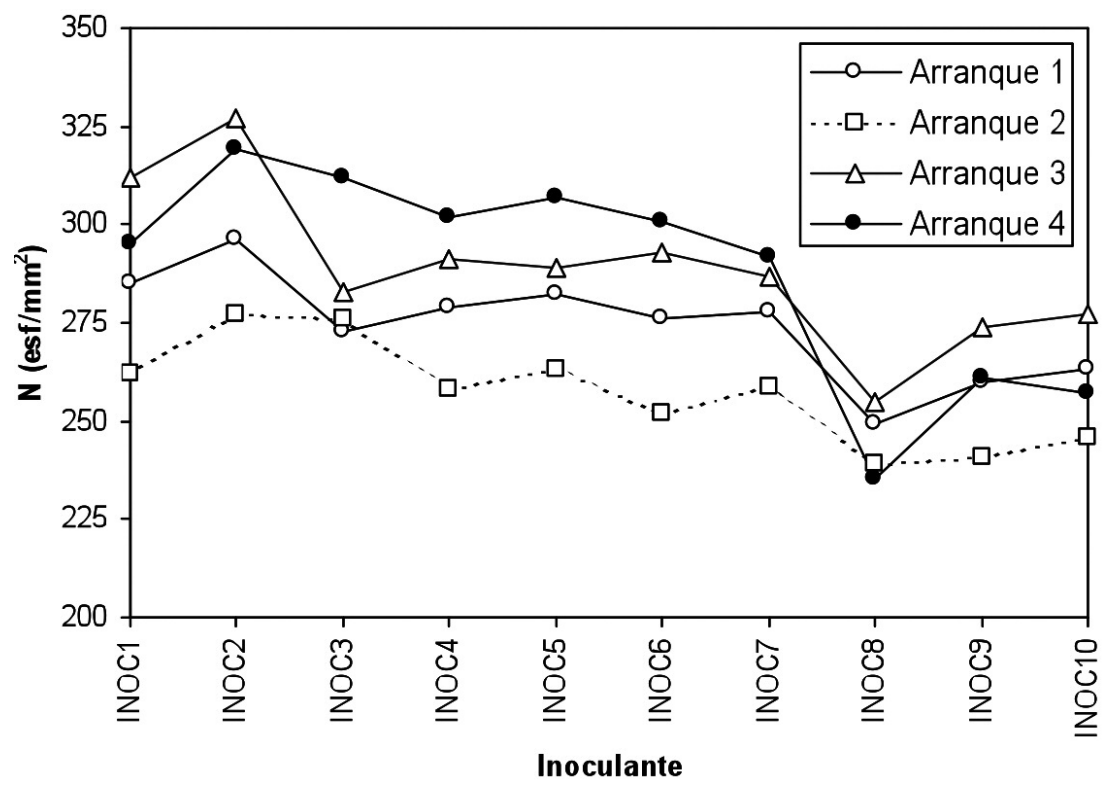

Figura 3. Evolución del parámetro $\mathrm{N}$ en las muestras inoculadas con diferentes inoculantes bajo condiciones de arranque de fabricación.

Figure 3. Evolution of the $N$ parameter in inoculated samples under starting conditions using different inoculants. 


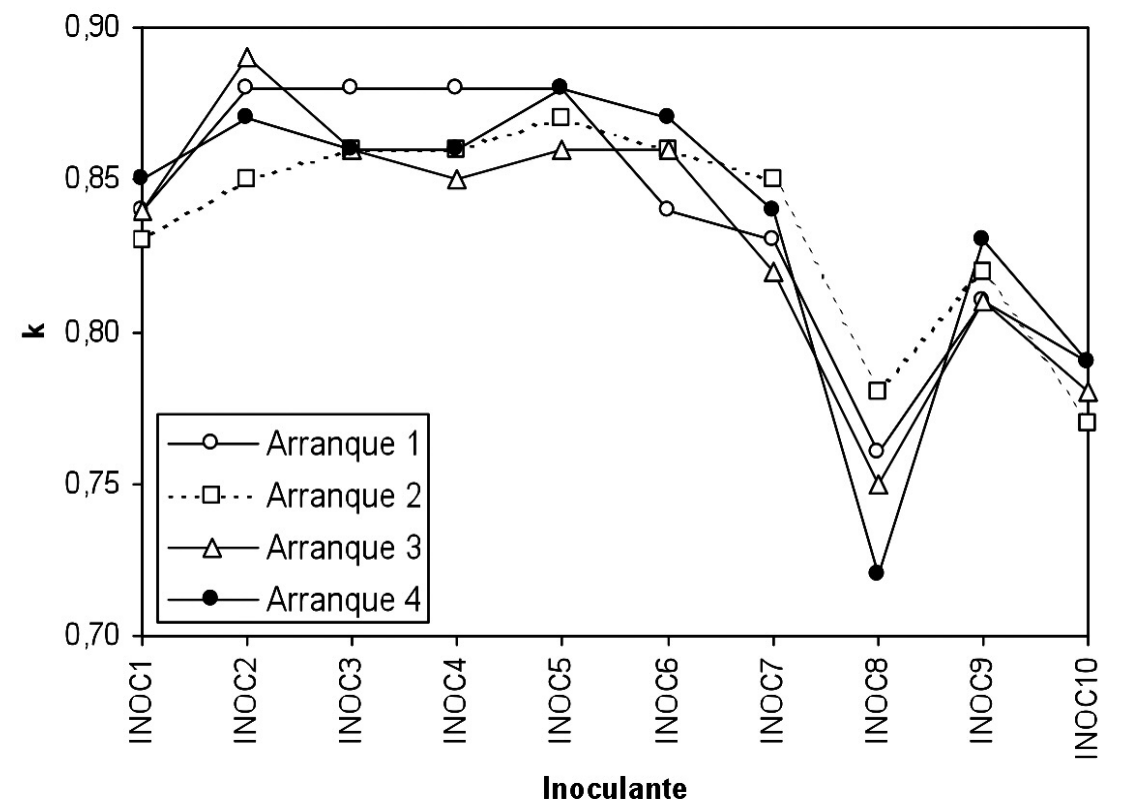

Figura 4. Evolución del parámetro k en las muestras inoculadas con diferentes inoculantes bajo condiciones de arranque de fabricación.

Figure 4. Evolution of the $k$ parameter in inoculated samples under starting conditions using different inoculants.

Tabla IV. Incidencia de la fase $\mathrm{Fe}_{3} \mathrm{C}$ en las cuñas de temple

Table IV. Fe ${ }_{3} \mathrm{C}$ incidence on the chilled specimens

\begin{tabular}{lrrrr}
\hline \multirow{2}{*}{ Inoculante } & \multicolumn{4}{c}{$\mathbf{H}(\mathbf{m m})$} \\
\cline { 2 - 5 } & Serie 1 & Serie 3 & Serie 11 & Serie 31 \\
\hline INOC1 & 5,7 & 4,3 & 6,8 & 5,5 \\
INOC2 & 6,0 & 4,0 & 5,8 & 5,4 \\
INOC3 & 6,2 & 5,3 & 7,4 & 6,6 \\
INOC4 & 7,4 & 4,8 & 6,0 & 5,7 \\
INOC5 & 6,0 & 5,0 & 6,4 & 5,3 \\
INOC6 & 7,1 & 5,2 & 7,0 & 5,5 \\
INOC7 & 8,2 & 5,0 & 7,2 & 5,5 \\
INOC8 & 14,8 & 11,7 & 10,4 & 10,6 \\
INOC9 & 15,2 & 8,1 & 10,6 & 8,7 \\
INOC10 & 9,0 & 8,2 & 8,0 & 6,2 \\
\hline
\end{tabular}

fabricación. Es necesario destacar que, en las muestras con valores de $\mathrm{k}$ más desfavorables, éstos son aún más bajos en los ensayos realizados en condiciones de arranque (Fig. 4).

Al examinar comparativamente los valores de $\mathrm{N}$ obtenidos en las muestras de metal inoculado con los 10 productos seleccionados, se observa un comportamiento análogo al detectado en los ensayos llevados a cabo bajo condiciones de arranque. Sistemáticamente, los productos INOC8, INOC9 y, en menor medida, el INOC10 dan lugar a los valores más reducidos de este parámetro, aunque el INOC3 también muestra el mismo comportamiento en 6 de las 8 series realizadas. En este último producto, los valores de $\mathrm{N}$ son comparables a los inoculantes con mejor respuesta, únicamente, cuando el metal de partida posee un elevado poder de nucleación grafítica (series 22 y 42). Los productos INOC1, INOC2, INOC4, INOC5, INOC6 e INOC7 proporcionan muestras de metal con poderes de nucleación comparables.

En el caso del parámetro k, su evolución es similar en todas las muestras inoculadas salvo en aquellas en las que se han utilizado los productos INOC8 e INOC10. Aunque con menos intensidad que en condiciones de arranque, ambos inoculantes dan lugar a las muestras de metal con mayor contracción secundaria durante el proceso de solidificación.

Al examinar la tabla IV se comprueba que el comportamiento de los inoculantes frente a la formación de carburos en el metal es similar bajo condiciones de arranque y fabricación normal. En este último caso, los productos que vuelven a originar los resultados más desfavorables son: INOC8, INOC9 e INOC10. 

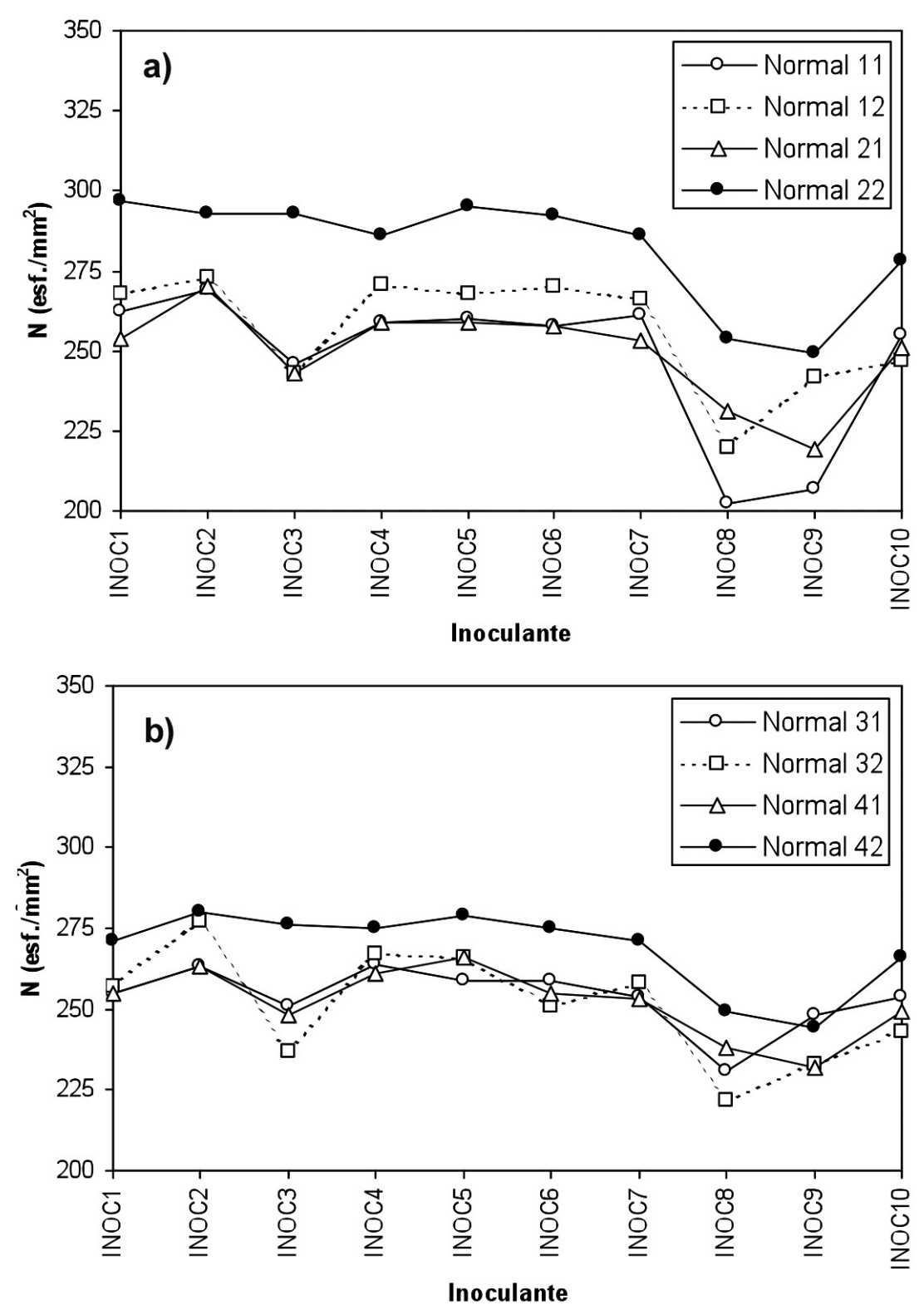

Figura 5. Evolución del parámetro $\mathrm{N}$ en las muestras inoculadas con diferentes inoculantes bajo condiciones normales de fabricación.

Figure 5. Evolution of the $N$ parameter in inoculated samples under normal conditions using different inoculants.

\subsection{Pruebas de fabricación sobre pieza real}

Teniendo en cuenta las dificultades a la hora de verificar sobre pieza la efectividad de todos los inoculantes incluidos en la tabla I, se han seleccionado 6 de ellos de acuerdo con los resultados descritos en los apartados 3.1 y 3.2. Los productos INOC2, INOC4, INOC5 e INOC6 corresponden a inoculantes con un buen comportamiento tanto en su poder de germinación grafítica como su capacidad para minimizar la tendencia del metal a la formación de microrrechupes. Por otra parte, se han seleccionado los productos INOC8 e INOC10 con el fin de verificar los resultados, comparativamente más desfavorables, obtenidos hasta el momento.

La tabla V contiene los resultados de la caracterización química y térmica del metal utilizado en cada una de las fabricaciones de piezas. También se incluyen los resultados obtenidos a partir de los análisis efectuados sobre dichas piezas. El orden de utilización de los inoculantes, fue el indicado en la tabla V. Los productos considerados con menor poder de nucleación (INOC8 e INOC10) originan densidades de 

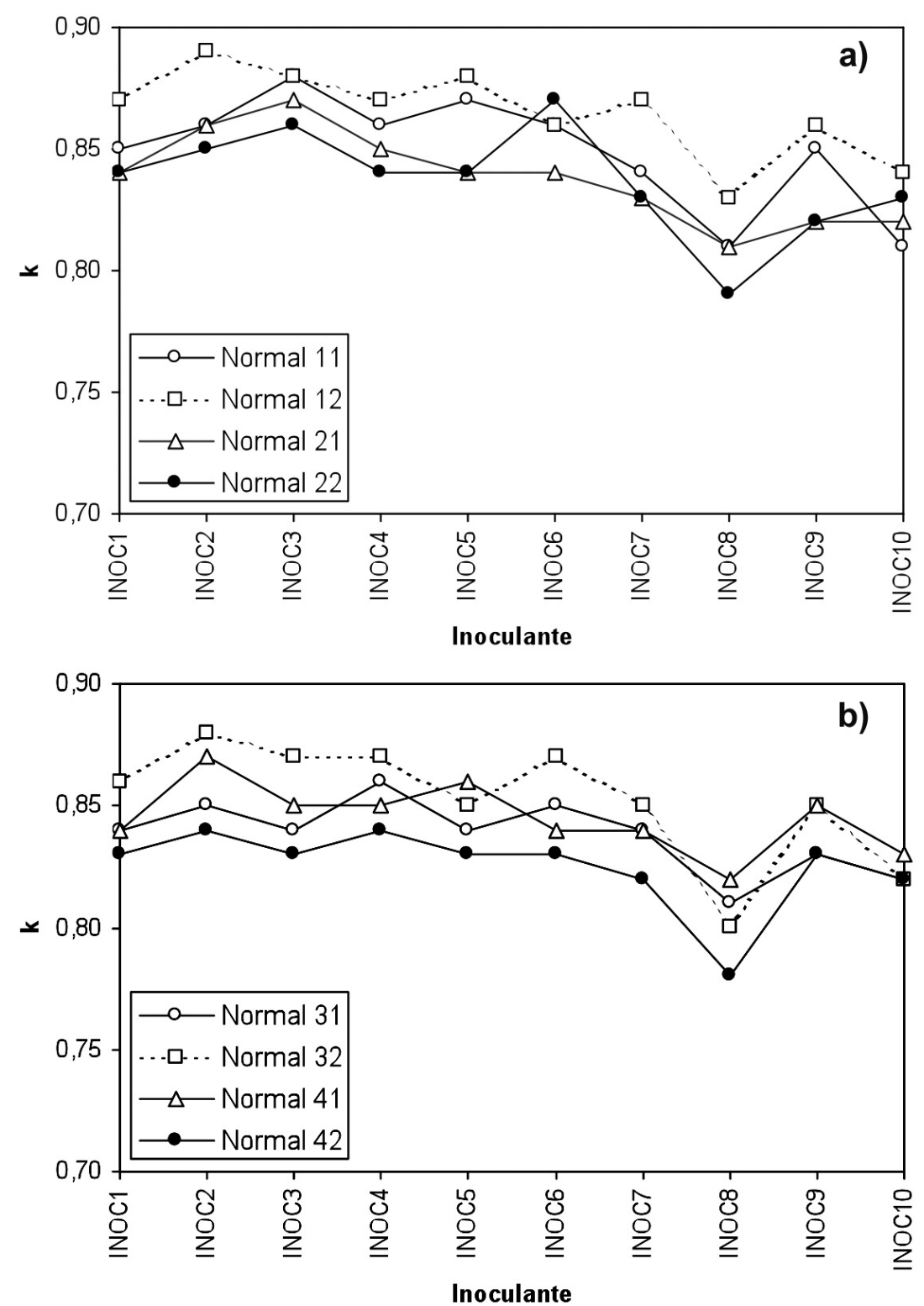

Figura 6. Evolución del parámetro $k$ en las muestras inoculadas con diferentes inoculantes bajo condiciones normales de fabricación.

Figure 6. Evolution of the $k$ parameter in inoculated samples under normal conditions using different inoculants.

esferoides inferiores en las piezas. En este caso, es necesario tener en cuenta que las diferencias entre los parámetros $\mathrm{N}$ y $\mathrm{D}$ se deben a los distintos módulos de enfriamiento entre la taza estándar de análisis térmico $(0,6 \mathrm{~cm})$ y la zona de la pieza donde se realizan las inspecciones metalográficas $(\sim 1 \mathrm{~cm})$.

Por otra parte, al analizar las piezas que muestran defecto se observa que existe una relación de los valores del parámetro k con el número y tamaño de los defectos. Los dos inoculantes seleccionados y con resultados más desfavorables en las series de ensayos descritas anteriormente (INOC8 e INOC10) originaron áreas de microrrechupe en todas las piezas inspeccionadas, siendo el volumen del defecto especialmente elevado cuando la inoculación del metal se llevó a cabo con el producto INOC8.

\section{DISCUSIÓN}

Partiendo de la necesidad de generar núcleos grafíticos a la hora de mejorar la maquinabilidad, homogeneizar la microestructura y optimizar las propiedades 
Tabla V. Características del metal y análisis de las piezas fabricadas

Table V. Characteristics of the melt and analysis of the manufactured castings

\begin{tabular}{|c|c|c|c|c|c|c|c|c|c|}
\hline \multirow{2}{*}{ Inoculante } & \multicolumn{6}{|c|}{ Características del metal } & \multicolumn{3}{|c|}{ Pieza (huella n 4) } \\
\hline & $\mathrm{C}(\%)$ & $\mathrm{Si}(\%)$ & $\operatorname{Mg}(\%)$ & $\mathbf{N}($ esf./mm²) & k & Total & $\mathrm{D}^{*}\left(\mathrm{esf} . / \mathrm{mm}^{2}\right)$ & Defecto & $\mathrm{V}^{* *}\left(\mathrm{~mm}^{3}\right)$ \\
\hline No inoculado & 3,71 & 2,52 & 0,032 & 135 & 0,69 & - & - & - & - \\
\hline INOC8 & 3,70 & 2,55 & 0,030 & 207 & 0,80 & 10 & 286 & 10 & 749 \\
\hline INOC10 & 3,70 & 2,58 & 0,028 & 209 & 0,79 & 10 & 299 & 10 & 264 \\
\hline INOC2 & 3,72 & 2,56 & 0,029 & 275 & 0,83 & 10 & 313 & 5 & 243 \\
\hline INOC4 & 3,71 & 2,55 & 0,030 & 256 & 0,83 & 10 & 307 & 8 & 282 \\
\hline INOC5 & 3,70 & 2,51 & 0,030 & 264 & 0,84 & 10 & 326 & 7 & 82 \\
\hline INOC6 & 3,72 & 2,58 & 0,027 & 252 & 0,83 & 10 & 318 & 5 & 160 \\
\hline
\end{tabular}

* Densidad de esferoides media obtenida en la zona donde aparece el defecto (3 piezas por inoculante).

** Volumen del defecto obtenido según la metodología descrita en la referencia [3].

mecánicas del hierro ${ }^{[18]}$, la búsqueda de substratos de gran efectividad que puedan desempeñar esta labor se convierte en uno de los principales objetivos en el diseño de inoculantes con aplicación industrial. Para ello, habitualmente, se parte de una aleación base FeSi en la que se adicionan pequeñas cantidades de elementos químicos considerados promotores de gérmenes nucleadores del grafito. Determinados autores establecen que estos elementos modifican la estructura cristalina exterior en partículas de óxidos y silicatos formados durante el tratamiento del metal con magnesio, de forma que los átomos de carbono puedan utilizarlos como punto de partida para formar grafito ${ }^{[19]}$.

En la bibliografía, parece haber coincidencia sobre la eficacia del silicio y calcio como elementos promotores de núcleos grafíticos y adicionalmente esferoidizante en el caso de este último ${ }^{[17}$ y 18$]$. Debido a la baja solubilidad del calcio en el hierro líquido, su capacidad para formar partículas de $\mathrm{CaO}$ y/o CaS es elevada, ejerciendo éstas de núcleos para el crecimiento del grafito cristalino durante el proceso de solidificación. Otros elementos considerados germinadores son el bismuto, lantano y cerio, aunque en el caso de estos dos últimos existen estudios contradictorios sobre su efecto beneficioso en la fabricación del hierro esferoidal ${ }^{[13]}$. En el caso del bario, determinados autores han descrito este elemento como un nucleador y estabilizador del grafito $^{[21]}$.

Los resultados del presente estudio parecen confirmar el efecto beneficioso del silicio y el calcio en los productos inoculantes seleccionados. La presencia de mayores contenidos de calcio (INOC4 e INOC5) conjuntamente con otros elementos (bismuto o zirconio) da lugar a resultados satisfactorios en todas las series de ensayos realizadas y piezas fabricadas. En el caso de los elementos lantano y cerio, ambos han demostrado actuar como promotores efectivos de nódulos grafíticos teniendo en cuenta el comportamiento exhibido por los productos INOC1 e INOC2. De este modo, el lantano no ha mostrado el efecto estabilizador de la fase cementita frente al grafito, promulgado por determinados autores ${ }^{[13]}$. La existencia de cantidades muy reducidas de azufre en el producto INOC2 origina resultados especialmente favorables en el comportamiento del metal durante la etapa de solidificación. Este hecho está en buen acuerdo con las observaciones realizadas por Lekakh y Loper ${ }^{[17]}$ o Skaland ${ }^{[19]}$ acerca de la influencia de los compuestos de azufre en la fabricación del hierro nodular.

Por otra parte, los peores comportamientos en relación tanto al poder de nucleación como la capacidad de contracción del metal se obtienen en aquellos inoculantes que contienen bario y/o manganeso en su composición (Tabla I). De forma sistemática, la presencia de bario junto con la ausencia de otros elementos considerados favorables hace que el producto INOC8 pueda considerarse el menos efectivo en base a los resultados obtenidos en el presente estudio. Aquellos inoculantes que, además de bario en su composición, contienen elementos como el La-TR (INOC6, INOC7 e INOC9), bismuto (INOC6 e INOC7), cerio (INOC6 e INOC9) y zirconio (INOC10) mejoran de forma importante su comportamiento respecto al producto INOC8, en el que, únicamente, el bario acompaña a los elementos habituales, silicio y calcio.

Teniendo en cuenta su capacidad para estabilizar carburos metálicos en bordes de grano, la presencia de Mn en los inoculantes comerciales INOC3, INOC8, INOC9 e INOC10 no parece adecuada en base a los resultados obtenidos en este estudio. El producto 
INOC3 es el único que contiene manganeso pero no bario, originando muestras de metal de colada con comportamientos más satisfactorios que el resto de los ya nombrados pero, en varias ocasiones, peores que los inoculantes exentos de estos dos elementos. También es necesario considerar que este inoculante contiene un $3 \%$ de zirconio como elemento germinador del grafito.

Respecto a los contenidos de aluminio, este elemento se conoce como un estabilizador del grafito, agente antiesferoidizante y promotor de porosidades de gas en el hierro esferoidal. Exceptuando el INOC5, los productos con mayores concentraciones de este elemento (INOC3, INOC8, INOC9 e INOC10) corresponden a los que han originado peores comportamientos en el metal. Sin embargo, la obtención de resultados favorables en el caso del producto INOC5 hace pensar que el intervalo de concentraciones de aluminio seleccionado en este trabajo no se puede considerar perjudicial en relación a la efectividad inoculadora del metal.

Al comparar los datos incluidos en la tabla III con los resultados mostrados en las figuras 3-5, se observa una relación entre el poder de nucleación del metal contenido en la unidad de colada y este mismo parámetro en las correspondientes series de muestras inoculadas. Es decir, cuanto mayor es el parámetro $\mathrm{N}$ en el metal no inoculado, se obtienen mayores valores tras el proceso de inoculación. Este comportamiento se observa para las series de ensayos realizadas tanto bajo condiciones de arranque como en las fabricaciones normales. De este modo y a pesar de la inoculación, la calidad del metal utilizado en la colada de los moldes está muy influida por el estado del metal contenido en la unidad de colada, el cual es, a su vez, consecuencia de anteriores etapas del proceso de fabricación (fusión y especialmente el tratamiento con el FeSiMg).

Sin embargo, al intentar relacionar la evolución del parámetro $\mathrm{N}$ en las series de arranque y las correspondientes realizadas bajo condiciones normales de fabricación, no ha sido posible encontrar una relación definida. La utilización de un determinado metal de arranque (inoculado o no) con un poder de nucleación más elevado no se traduce necesariamente en la obtención de un metal con mayores valores del parámetro $\mathrm{N} \mathrm{du}$ rante la fabricación normal posterior. Este hecho puede considerarse el resultado de la influencia que la metodología de fusión (metal base) y el tratamiento posterior con FeSiMg tienen sobre la presencia de gérmenes grafíticos en el metal líquido. Por tanto, cada cuchara tratada define en gran medida las características del metal contenido en la unidad de colada e influye en la calidad del metal inoculado. Un ejemplo de este hecho es la serie 22, en la que se obtienen muestras de metal con valores del parámetro $\mathrm{N}$ comparativamente más elevados. La explicación se encuentra en la adición de 12 kg de FeSi realizada en la cuchara de tratamiento con el fin de ajustar el contenido de silicio en el metal vertido desde el horno de fusión.

Aunque se trata de un aspecto bien conocido, se confirma el hecho de que los inoculantes que aportan un menor poder de nucleación al metal son, también, los que menos capacidad muestran a la hora de evitar la formación de cementita en condiciones de enfriamiento crítico (Tabla IV). Por otra parte, no se observa que la tendencia a la formación de la fase metaestable sea mayor en el metal preparado en los arranques que en el utilizado en condiciones de fabricación normal. Probablemente, dicho comportamiento está relacionado con el aumento en la adición de inoculante sobre el metal en condiciones de arranque $(0,20 \%)$.

En relación a la capacidad de contracción en la etapa final de la solidificación se observa que, tanto en las series de arranque como en las realizadas en condiciones normales de fabricación, la evolución del parámetro $\mathrm{k}$ al inocular el metal no parece depender de la capacidad de contracción mostrada por el metal contenido en la unidad de colada. Aunque en estudios anteriores se ha establecido una relación entre los parámetros $\mathrm{N} \mathrm{y}^{[3 \mathrm{y} 6]}$, ésta se manifiesta, principalmente, cuando se comparan muestras de metal sin y con inoculación (este proceso aumenta el valor tanto de $\mathrm{N}$ como de k, tal y como ocurre en este estudio). Sin embargo, es posible obtener muestras de metal inoculado con poderes de nucleación similares pero con diferentes valores de $\mathrm{k}$ o viceversa.

En comparación con las muestras obtenidas en condiciones normales de fabricación, las pertenecientes a los arranques originan valores de $\mathrm{k}$ más bajos cuando se utilizan los inoculantes menos efectivos (Figs. 4 y 6 ). Este hecho se relaciona con una mayor capacidad de contracción en el metal no inoculado y elaborado en los arranques (tabla III), junto con la menor efectividad relativa de los inoculantes que muestran un peor comportamiento. En el caso de los productos más efectivos, se obtienen valores del parámetro k que son comparables a los obtenidos en condiciones normales de fabricación.

\section{CONCLUSIONES}

La utilización de un sistema predictivo basado en técnicas de análisis térmico permite el control rápido y eficaz de las características del metal empleado en planta. En el presente trabajo, uno de estos sistemas se ha aplicado satisfactoriamente con el fin de estudiar la influencia de diferentes inoculantes sobre el metal de colada bajo condiciones reales de fabricación. Las principales conclusiones obtenidas son: 
El tratamiento de esferoidización afecta de manera importante al poder de nucleación del metal contenido en la unidad de colada y su capacidad de contracción secundaria. De este modo, la calidad del metal de colada puede sufrir variaciones de unas cucharas a otras, haciendo necesario un control por cada cuchara introducida en el sistema.

Bajo condiciones de arranque de fabricación, el metal contenido en el dispositivo de colada muestra un poder de nucleación menor y una capacidad de contracción mayor que en las fabricaciones habituales. La inoculación del metal con un inoculante efectivo mejora sus características en ambas condiciones de trabajo.

El aumento en la adición de inoculante de 0,15 a $0,20 \%$ en condiciones de arranque de fabricación ha mostrado ser una medida eficaz a la hora de reparar la degradación de los gérmenes grafíticos en el metal tratado con mayores cantidades de FeSiMg.

Se observa la relación ya descrita en la bibliografía entre los parámetros $\mathrm{N}$ y k cuando se inocula el metal, es decir, el poder de nucleación es mayor y disminuye la capacidad de contracción (aumenta el valor de k). En condiciones de arranque, el factor $\mathrm{k}$ parece seguir una evolución más crítica cuando el producto inoculante utilizado muestra una peor efectividad.

La presencia de mayores contenidos de silicio y calcio en los inoculantes produce resultados satisfactorios en relación al poder de nucleación, la capacidad de contracción y formación de cementita del metal tanto en condiciones de arranque como en fabricación normal. Este mismo efecto puede ser asignado a la utilización de bismuto, lantano, cerio y/o zirconio.

En el caso del producto INOC2, en el cual se ha detectado el mayor contenido de azufre en su composición química, los resultados obtenidos son comparativamente más positivos, en todos los aspectos metalúrgicos considerados.

Los inoculantes que contienen bario y manganeso en su composición muestran una peor efectividad cuanto menor es su contenido en elementos favorables a la nucleación del grafito, es decir, silicio, calcio, bismuto, lantano, cerio y/o zirconio. El caso más crítico lo constituye el producto INOC8.

Las pruebas de fabricación efectuadas con piezas reales confirman que los inoculantes que alcanzan menores valores de $\mathrm{N}$ y $\mathrm{k}$ en las muestras estándar originan microrrechupes de mayor tamaño en dichas piezas.

\section{Agradecimientos}

Los autores de este trabajo agradecen a la fundición Betsaide, S.A.L. por la financiación y la colaboración prestada por su personal de planta a la hora de realizar los ensayos experimentales. Este trabajo se ha realizado en el marco del proyecto PROFIT 2005 con referencia CIT 020600-2005-28, perteneciente al Ministerio de Educación y Ciencia del Gobierno de España.

\section{REFERENCIAS}

[1] I.G. Chen y D.M. Stefanescu, AFS Trans. 92 (1984) 947-964.

[2] Y.X. Li, X.R. Xu, Q. Wang y B.C. Liu, Int. J. Cast Met. Res. 16 (2003) 41-45.

[3] P. Larrañaga, J. M. Gutiérrez, A. Loizaga, J. Sertucha y R. Suárez, AFS Trans. 112 (2008) 547561.

[4] J. Tinoco, P. Delvasto, O. Quintero y H. Fredriksson, Int. J. Cast Met. Res. 16 (2003) 53-58.

[5] C. Labrecque y M. Gagné, AFS Trans. 106 (1998) 83-90.

[6] J. Sertucha, R. Suárez, J. Izaga, L.A. Hurtado y J. Legazpi, Int. J. Cast Met. Res. 19 (2006) 315322.

[7] T. Skaland y $\varnothing$. Grong, AFS Trans. 99 (1991) 153-157.

[8] J. Lacaze, Acta Mater. 47 (1999) 3.779-3.792.

[9] L. Battezzati, M. Baricco, C.A. Coria, G. Serramoglia y F. Marongiu, Int. J. Cast Met. Res. 16 (2003) 87-92.

[10] S. Jung, T. Ishikawa y H. Nakae, Mater. Sci. Eng. A 476 (2008) 350-356.

[11] C.R. Loper y K. Fang, AFS Trans. 112 (2008) 673-682.

[12] P. Larrañaga, J. Sertucha y R. Suárez, Rev. Metal. Madrid 42 (2006) 244-255.

[13] M.I. Onsøien, $\varnothing$. Grong, T. Skaland y S.O. Olsen, AFS Trans. 105 (1997) 147-152.

[14] A. Louvo, E. Alhainen Pellikka y P. Eklund, AFS Trans. 99 (1991) 237-244.

[15] C.A.S. Ribeiro, D. Santos, W. Baumgart, F. Vilela y C. Henke, Int. J. Cast Met. Res. 20 (2003) 4752.

[16] I. Asenjo, P. Larrañaga, J. Sertucha, R. Suárez, J.M. Gómez, I. Ferrer y J. Lacaze, Int. J. Cast Met. Res. 20 (2007) 319-324.

[17] S. Lekakh y C.R. Loper, AFS Trans. 111 (2003) paper 03-103.

[18] S.O. Olsen, T. Skaland y C. Hartung, Proc. 66th World Foundry Congress, Estambul, Turquía, 2004, pp. 891-902.

[19] T. Skaland, Tesis Doctoral, Metallurgisk Institutt, Trondheim, Norway, 1992.

[20] A. Loizaga, J. Sertucha y R. Suárez, Rev. Metal. Madrid 44 (2008) 432-446.

[21] J. F. Wallace y R.D. Maier, Proc. AFS-CMI Conf., Rosemont, Illinois, EE. UU., 1979, pp. 41-73. 